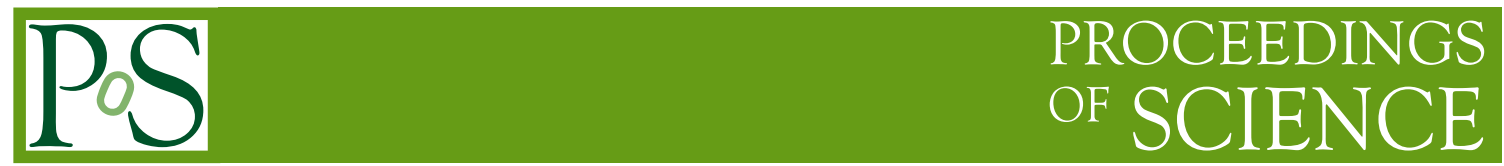

\title{
Beyond Standard Model Searches at LHC
}

\author{
Jike Wang ${ }^{* \dagger}$ \\ DESY \\ E-mail: jike.wang@desy.de
}

In year 2015, ATLAS and CMS experiments have collected up to $3 \mathrm{fb}^{-1} \mathrm{pp}$ collision data with center-of-mass energy at $13 \mathrm{TeV}$. Many interesting new Beyond Standard Model (BSM) results have come out with this unprecedented high energy. They will be presented in this article.

Flavor Physics and CP Violation,

6-9 June 2016

Caltech, Pasadena CA, USA

* Speaker.

${ }^{\dagger}$ A footnote may follow. 


\section{Searches for high mass di-boson and di-photon resonances}

There was a large excess seen in ATLAS Run-I di-boson resonance searches (mostly from the $V V \rightarrow J J$ channel). Although not as significant as in ATLAS, an excess was also observed by CMS in several channels in the same mass range [1]. It would be very interesting to check these excesses again in the new $13 \mathrm{TeV}$ collision data.

In these high mass searching channels, when $W / Z$ bosons decay hadronically, they normally form as a single large $-R$ jet $(J)$. And jet-substructure techniques are used to identify the large $-R$ jet to be $W / Z$. As examples, Figure 1(a) shows the ATLAS $X \rightarrow V V \rightarrow v v J$ channel; Figure 1(b) shows the CMS $X \rightarrow V V \rightarrow l v J$ channel. In the $X \rightarrow V V \rightarrow v v J$ channel, the discriminating variable in the event is the transverse mass between the missing transverse momentum $\left(E_{\mathrm{T}}^{\text {miss }}\right)$ and the large $-R$ jet. There is also the large $E_{\mathrm{T}}^{\text {miss }}$ requirement for this channel: $E_{\mathrm{T}}^{\text {miss }}>250 \mathrm{GeV}$. Though the resolution of the discriminating variable is not very good, but actually this channel is a quite sensitive channel in the high mass region, due to the big signal acceptance. In the $X \rightarrow V V \rightarrow$ $l v J$ channel, $W$ mass constraint method is used to obtain neutrino $p_{z}$.

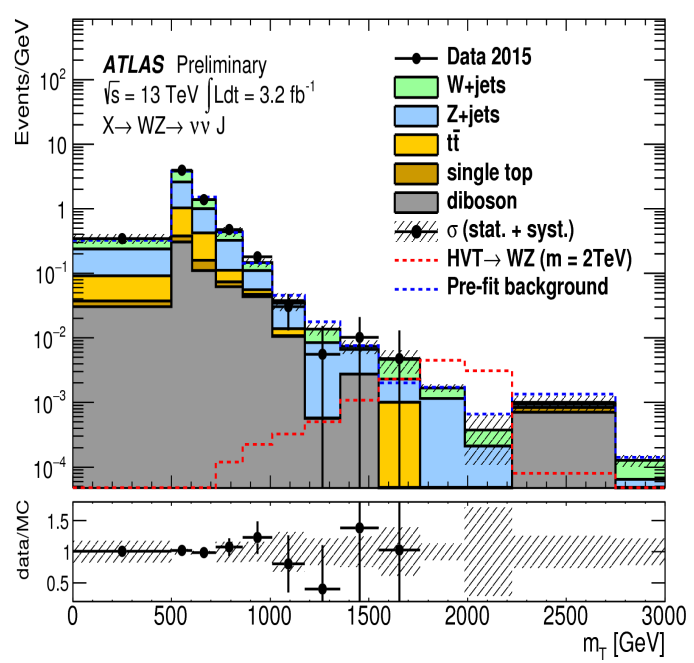

(a)

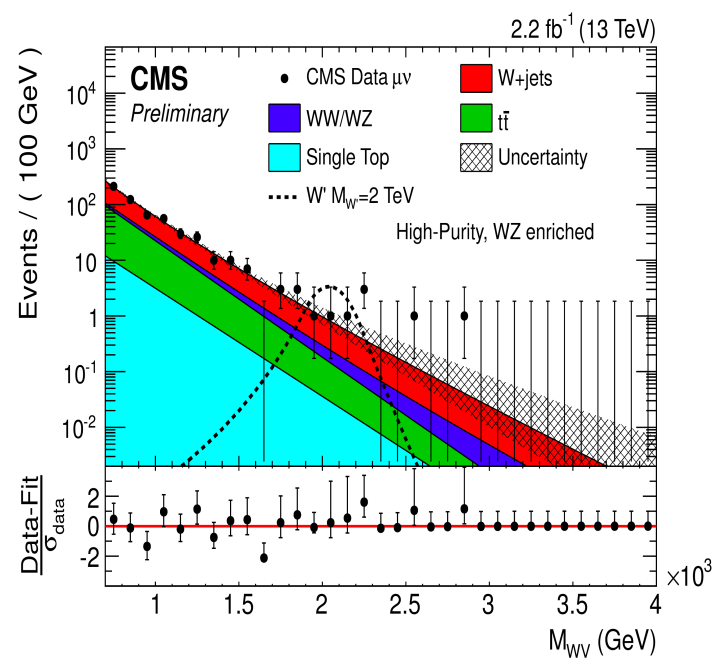

(b)

Figure 1: $X \rightarrow V V \rightarrow v v J$ and $X \rightarrow V V \rightarrow l v J$ channels.

Figure 2 shows the ATLAS and CMS combination results of the di-boson resonance searching channels, respectively. For the $201513 \mathrm{TeV}$ collision data, no clear excess is observed so far in both experiments, and similar sensitivity has been achieved between ATLAS and CMS.

In the $201513 \mathrm{TeV}$ collision data, both ATLAS and CMS have observed an interesting resonant bump around $750 \mathrm{GeV}$ [3], in the di-photon mass spectrum. ATLAS has two selection sets for this search, one is aiming for spin- 0 analysis, which is optimized for Higgs-like signals. There are about 2900 data events are left after spin-0 selections (for mass $>200 \mathrm{GeV}$ ); the other is dedicated for spin- 2 analysis, which preserves more events in the high mass region (there are about 5100 data events are left for mass $>200 \mathrm{GeV}$ ). Figure 3 shows the di-photon mass distributions for spin- 0 selections and spin-2 selections, respectively. The two dimension (2D) significance is shown in 

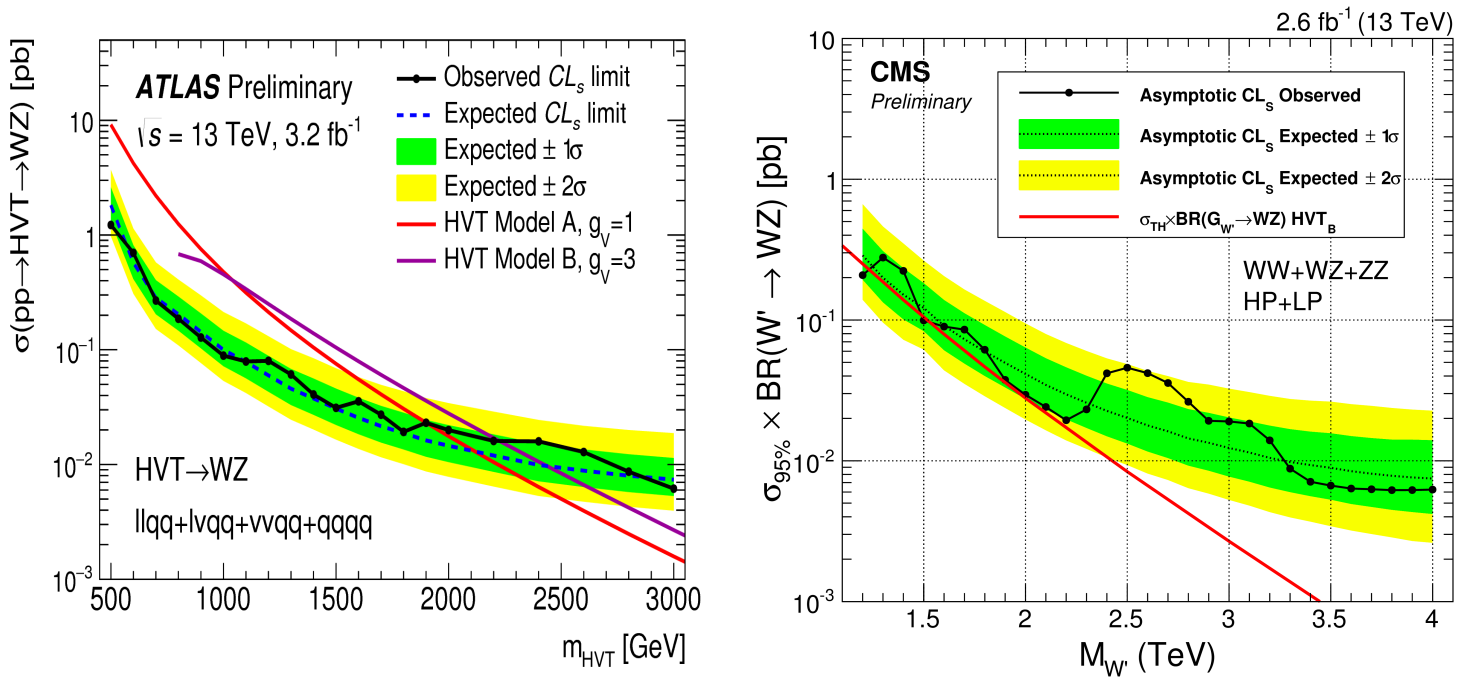

Figure 2: The combination results of the di-boson resonance searching channels.

Figure 4. From the two plots, for both spin- 0 and spin- 2 selections, the largest local significance is at mass about $750 \mathrm{GeV}$ and width about $45 \mathrm{GeV}$. The largest global significance is about $2 \sigma$ for both cases. The significance of the CMS, with different width assumptions, is shown in Figure 5.
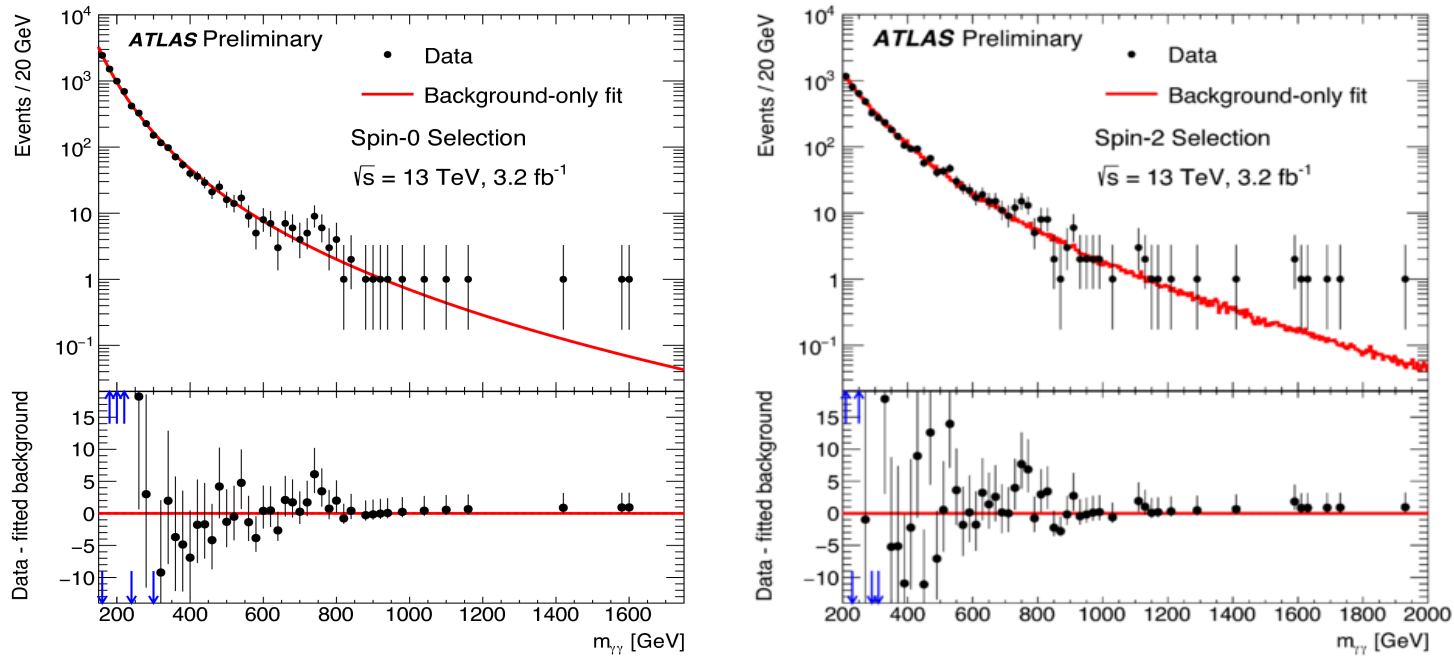

Figure 3: The di-photon mass distributions for spin- 0 selections and spin- 2 selections, in the ATLAS experiment.

\section{Searches for charged Higgs boson}

Charged Higgs bosons produced in association with a single top quark and decaying via $H^{ \pm} \rightarrow$ $\tau \nu$ are searched for with the ATLAS experiment at the LHC, using proton-proton collision data at $\sqrt{s}=13 \mathrm{TeV}$ corresponding to an integrated luminosity of $3.2 \mathrm{fb}^{-1}$. The final state is characterised by the presence of a hadronic $\tau$ decay and missing transverse momentum, as well as a hadronically 

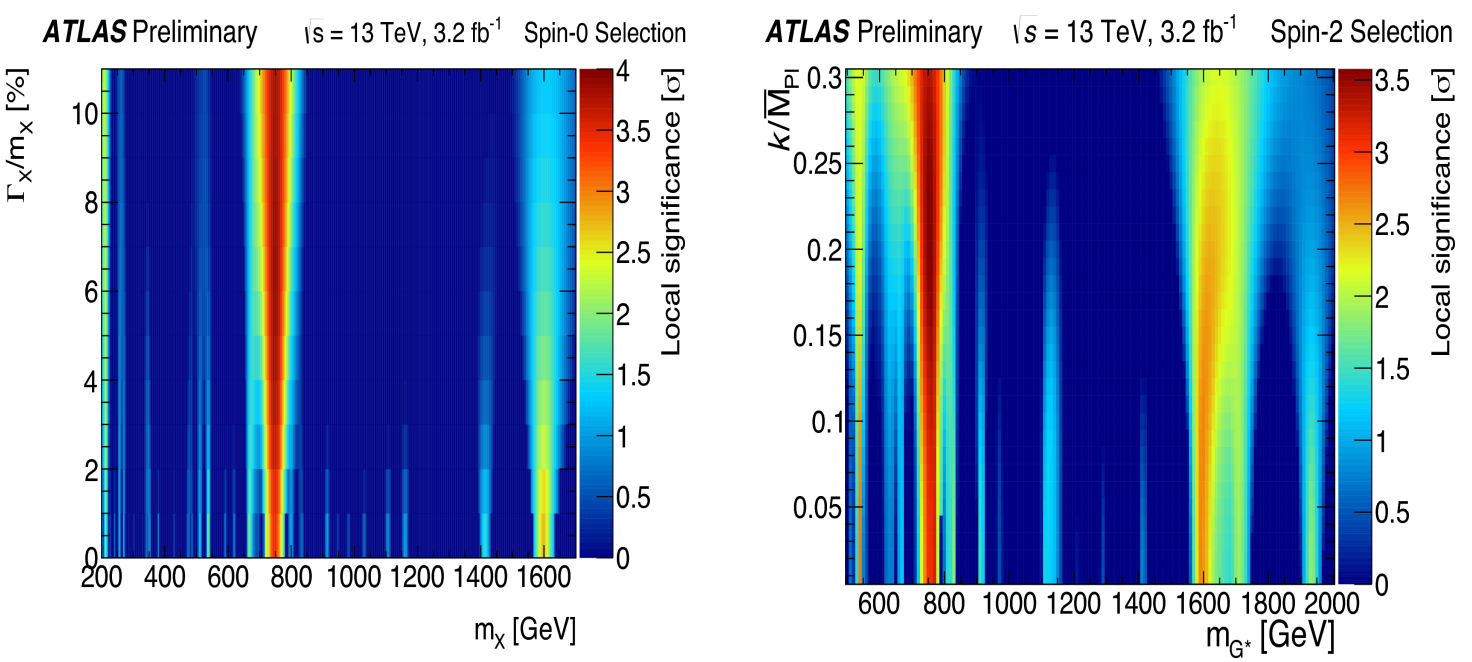

Figure 4: The 2D local significance for spin-0 selections and spin-2 selections, in the ATLAS experiment.
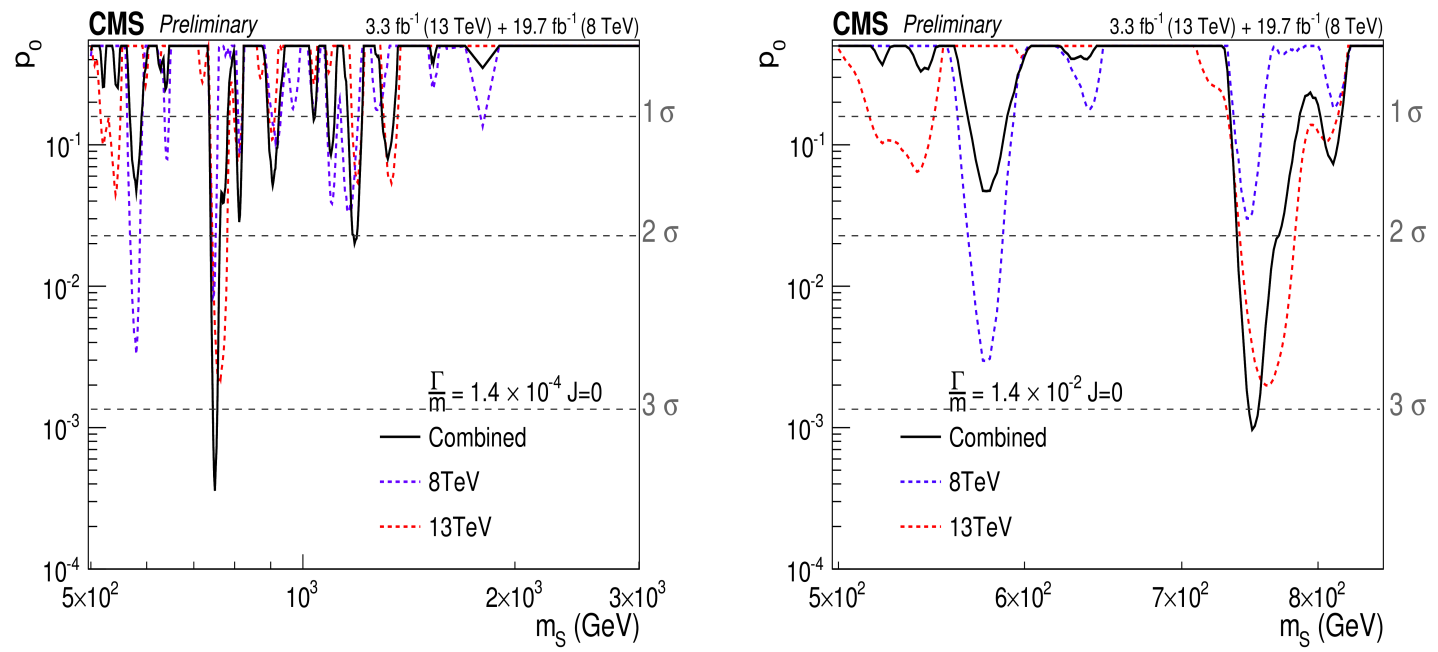

Figure 5: The significance for different width signals, in the CMS experiment.

decaying top quark, resulting in the absence of high-transverse-momentum electrons and muons $\left(p p \rightarrow[b] t H^{+} \rightarrow[b](j j b)\left(\tau_{\text {had }} v\right)\right.$ ). The leading-order Feynman diagrams for the production of a charged Higgs boson with a mass $m_{H^{+}}>m_{t o p}$, in association with a single top quark, are shown in Figure 6.

For the selected events, the transverse mass $m_{\mathrm{T}}$ of the $\tau_{\text {had-vis }}$ and $E_{\mathrm{T}}^{\text {miss }}$ system is defined as:

$$
m_{\mathrm{T}}=\sqrt{2 p_{\mathrm{T}}^{\tau} E_{\mathrm{T}}^{\mathrm{miss}}\left(1-\cos \Delta \phi_{\tau, \mathrm{miss}}\right)}
$$

The $m_{\mathrm{T}}$ after full event selection and a fit to the data with the background-only hypothesis is shown in Figure 7. Two signals are shown on the plot.

Figure 8 shows the $95 \%$ CL exclusion limits on $\tan \beta$ as a function of $m_{H^{+}}$in the context of the hMSSM, compared with the ATLAS Run 1 results. Values of $\tan \beta$ in the range $42-60$ are excluded 

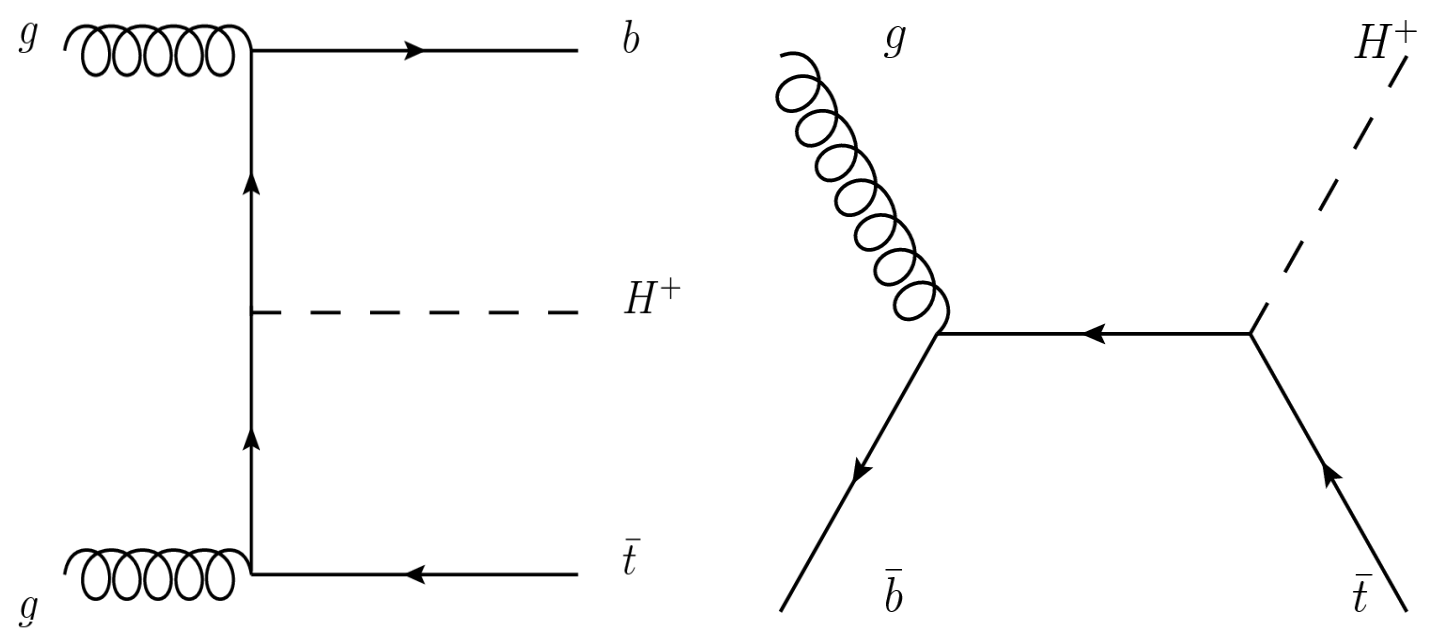

Figure 6: Leading-order Feynman diagrams for the production of a charged Higgs boson with a mass $m_{H^{+}}>m_{t o p}$, in association with a single top quark (left in the 4FS, and right in the 5FS).

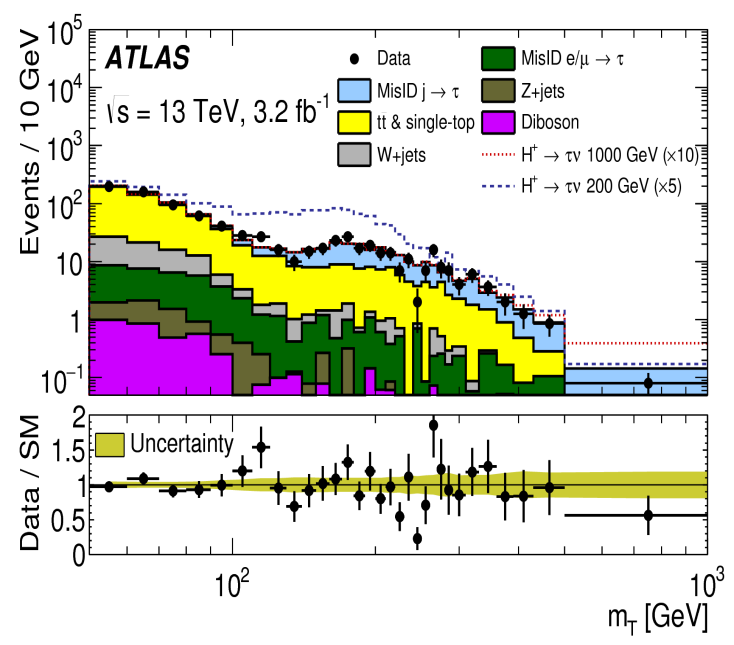

Figure 7: Distribution of $m_{\mathrm{T}}$ after full event selection and a fit to the data with the background-only hypothesis. The horizontal axis starts at $m_{\mathrm{T}}=50 \mathrm{GeV}$ and is in logarithmic scale. Two $\mathrm{H}^{+}$signal hypotheses are included on the stack. The signal sample at 200 (1000) $\mathrm{GeV}$ is scaled to 5 (10) times the cross section predicted at $\tan \beta=60$ in the hMSSM benchmark scenario. Bins are $10 \mathrm{GeV}$ in width up to $310 \mathrm{GeV}$ and then have a varying size. The total (statistical and systematic) uncertainties in the SM prediction are shown in the lower plot. 
for a charged Higgs boson mass of $200 \mathrm{GeV}$. At $\tan \beta=60$, above which no reliable theoretical calculations exist, the $\mathrm{H}^{+}$mass range from 200 to $340 \mathrm{GeV}$ is excluded. The limits of this search surpass those obtained with the $p p$ collision data at $\sqrt{s}=8 \mathrm{TeV}$.

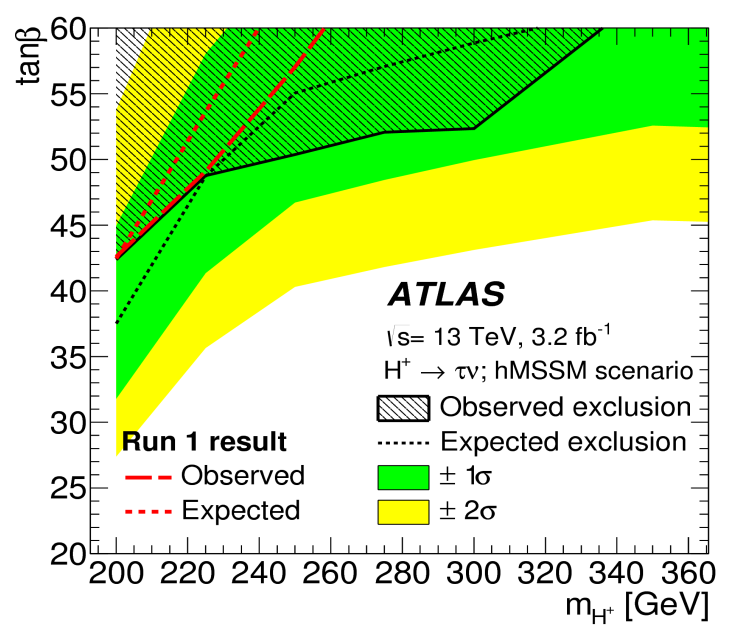

Figure 8: $95 \% \mathrm{CL}$ exclusion limits on $\tan \beta$ as a function of $m_{H^{+}}$, shown in the context of the hMSSM, for the regions in which reliable theoretical calculations exist $(\tan \beta \leq 60)$. As a comparison, the red curves show the observed and expected exclusion limits from Run 1 analyses of $p p$ collisions measured at $\sqrt{s}=8$ TeV by ATLAS.

\section{Search for higgs-pair production}

For higgs-pair production, there are two modes: non-resonant and resonant. The non-resonant production exists in SM, but the predicted cross-section is very small, a large measured event rate would clearly indicate new physics. The resonant production is predicted in many BSM models, e.g. 2HDM, Hidden-sector etc. The corresponding Feynman diagrams are shown in Figure 9.

ATLAS has released the results of two higgs-pair channels based on the $13 \mathrm{TeV}$ data collected in 2015. One is the $\mathrm{hh} \rightarrow \mathrm{bb} \gamma \gamma$ channel. It has very low production cross-section, but there is very good mass resolution. $\mathrm{X} \rightarrow \mathrm{hh} \rightarrow \mathrm{bb} \gamma \gamma$ is mainly sensitive in the low mass region. The other is $\mathrm{hh} \rightarrow$ bbbb channel. It has the largest cross-section in all the higgs-pair production channels, but there is very big background. $\mathrm{X} \rightarrow \mathrm{hh} \rightarrow$ bbbb is mainly sensitive in the high mass region. CMS has also released the results of $\mathrm{hh} \rightarrow \mathrm{bb} \tau \tau, \mathrm{hh} \rightarrow$ bbWW channels based on the $13 \mathrm{TeV}$ data.

For the ATLAS $\mathrm{X} \rightarrow \mathrm{hh} \rightarrow \mathrm{bbbb}$ analysis, there are both resolved and boosted analysis. The dominated background in the signal region is the multi-jets production, which is data-driven from control region. Figure 10 shows the final 4-body invariant mass distributions of the resolved and boosted analysis, respectively. The combined expected and observed upper limit for $\mathrm{pp} \rightarrow \mathrm{H} \rightarrow \mathrm{hh} \rightarrow \mathrm{bbbb}$ with fixed $\Gamma_{H}=1 \mathrm{GeV}$, at the 95\% confidence level is shown in Figure 11.

For the CMS $\mathrm{X} \rightarrow \mathrm{hh} \rightarrow \mathrm{bb} \tau \tau$ analysis, both non-resonant and resonant analysis have been covered. Then further split into $\mu \tau_{h}, e \tau_{h}$ and $\tau_{h} \tau_{h}$ channels, according to the decaying mode of $\tau$. The non-resonant analysis has tried to measure the higgs self-couplings through the trilinear 


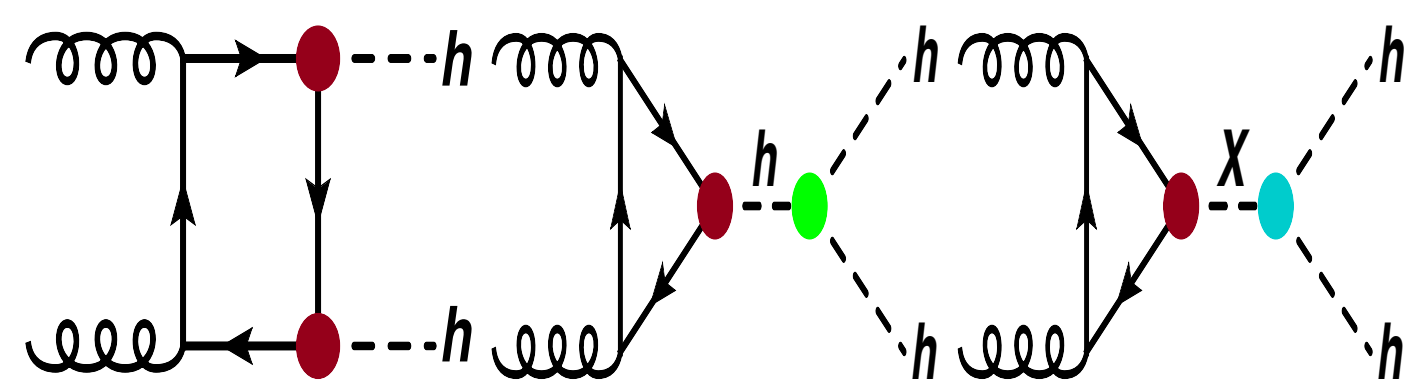

(a)

(b)

(c)

Figure 9: Leading-order production modes for Higgs boson pairs in the SM through (a) a heavy-quark loop and (b) the Higgs self-coupling. The total SM contribution is the sum of the two modes, which includes significant destructive interference. BSM Higgs boson pair production could proceed through changes in the SM Higgs couplings in (a) and (b), or through (c) an intermediate resonance, $X$.

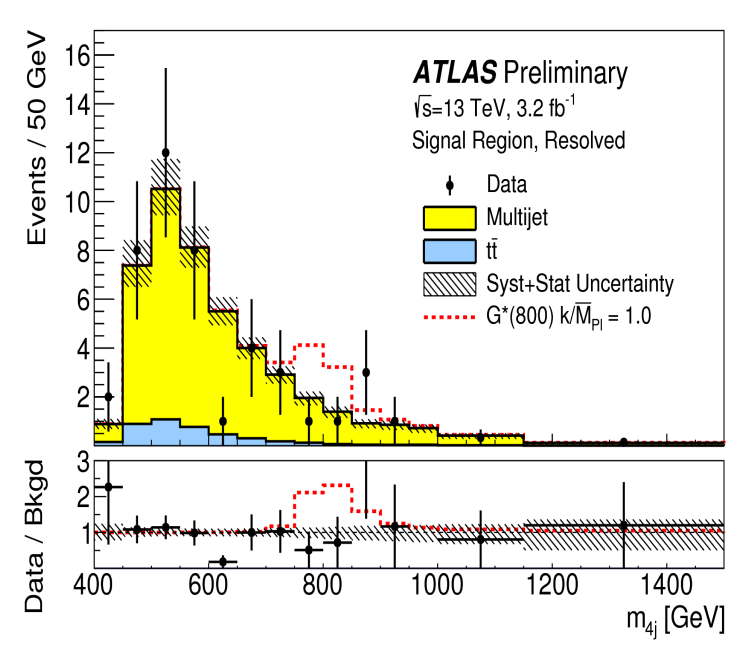

(a)

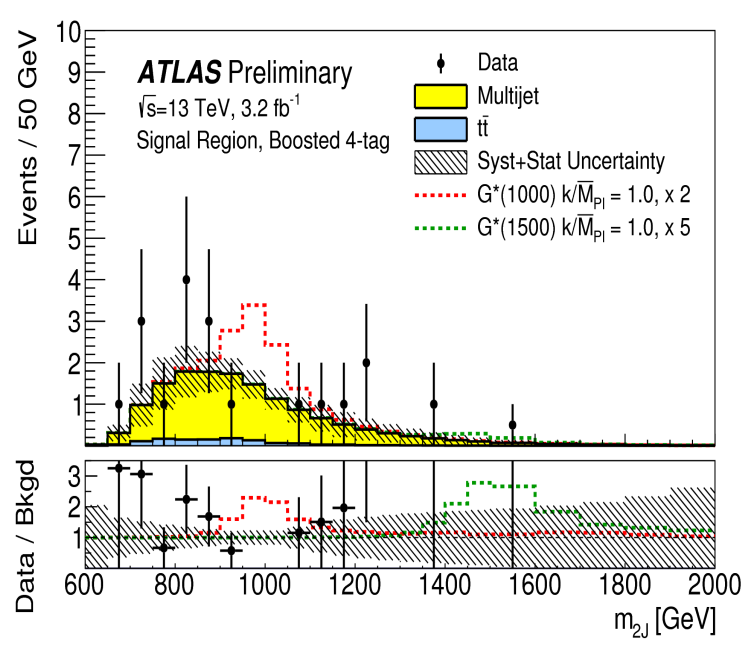

(b)

Figure 10: Final 4-body invariant mass distributions of the resolved (left) and boosted analysis (right).

Higgs boson coupling $\left(\lambda_{\text {hhh }}\right)$. In the absence of evidence for a signal in the non-resonant analysis, the 95\% CL upper limits on cross section times branching ratio for the non-resonant Higgs boson pair production, as a function of the ratio of the anomalous trilinear coupling to the SM trilinear coupling $\left(\kappa_{\lambda}=\lambda_{\text {hhh }} / \lambda_{\text {hhh }}^{\mathrm{SM}}\right)$, have been provided as shown in Figure 12(a).

In the absence of evidence for a signal in the resonant analysis, the $95 \%$ CL upper limits on cross section times branching ratio for the resonant Higgs boson pair production, as a function of the mass of the resonance $m_{H}$. These limits are shown in Figure 12(b) for the combination of the three final states. The results presented here are computed using the asymptotic approximation [5]. The observed (expected) limits on $\sigma(\mathrm{pp} \rightarrow \mathrm{H}) \times \mathrm{BR}(\mathrm{H} \rightarrow \mathrm{hh} \rightarrow \mathrm{bb} \tau \tau)$ range from $O(1)$ 


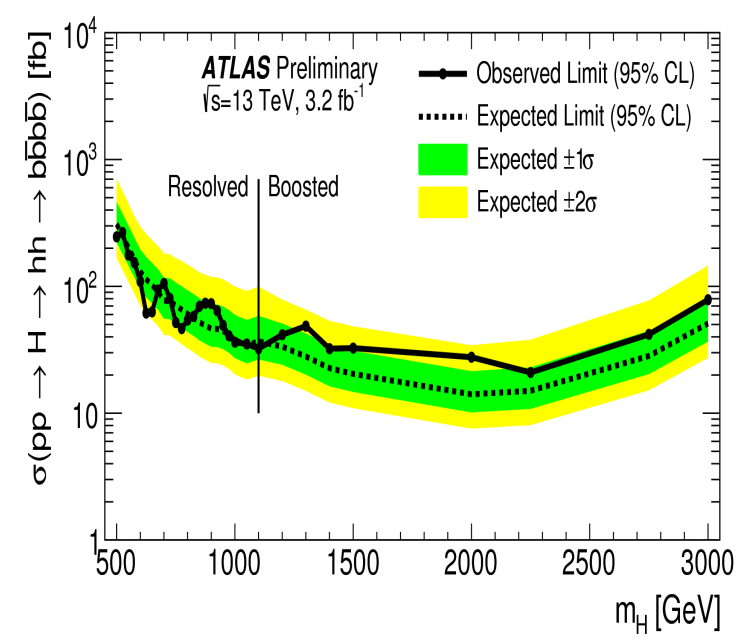

Figure 11: The combined expected and observed upper limit for $\mathrm{pp} \rightarrow \mathrm{H} \rightarrow \mathrm{hh} \rightarrow$ bbbb with fixed $\Gamma_{H}=1$ $\mathrm{GeV}$.

$\mathrm{pb}$ to $O\left(10^{-2}\right) \mathrm{pb}$. As expected, the value of the excluded cross section decreases as $m_{H}$ increases.

\section{Search for dark matter}

Dark matter (DM) production at colliders is characterized by missing transverse energy from DM particles escaping the detector and recoiling against a visible final state $X$ (mono- $X$ ). Various mono- $X$ searching channels have been carried at ATLAS and CMS. Here $X$ could be a hadronic jet, a photon or a $W / Z$ boson. There are generally two models for DM interpretation at LHC, one is an effective field theory (EFT), the other is a simplified model which includes a mediator. The Feynman diagrams of the two models are shown in Figure 13. With some assumptions of the simplified model, all the mono- $X$ channels and the di-jet search can be put together into the summary plot 14 .

The discovery of the higgs boson opens a new collider probe of dark matter: mono-higgs, which would probe directly the structure of the effective DM-SM coupling (different from other Mono- $X)$. There are three mono-higgs channels: mono-higgs $(\rightarrow b b)$, mono-higgs $(\rightarrow \gamma \gamma)$ and mono-higgs $(\rightarrow 4 l)$. mono-higgs $(\rightarrow b b)$ has the best sensitivity. Though it has huge background, but it has much bigger signal acceptance. The interpretation plots of the ATLAS mono-higgs $(\rightarrow b b)$ are shown in Figure 15.

\section{Search for super symmetric particles}

SUSY model predicts that each SM particle has a super-symmetric partner, and differs only by $1 / 2$ of spin. For example, Figure 16 shows the SM particles and their super symmetric particles. There are several advantages of SUSY model. For example it could unify gauge couplings, provide possible solution to hierarchy problem; Some SUSY particles are also good dark matter candidate. There are extremely large SUSY phase-spaces to probe at LHC. 


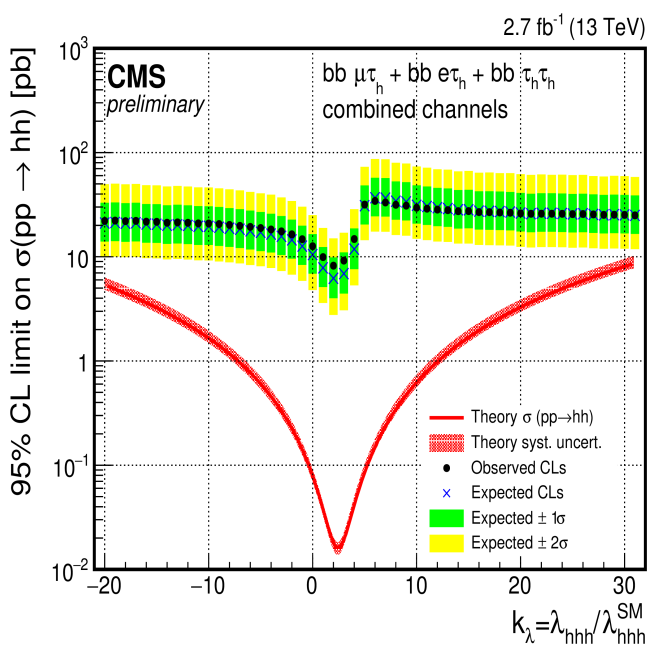

(a)

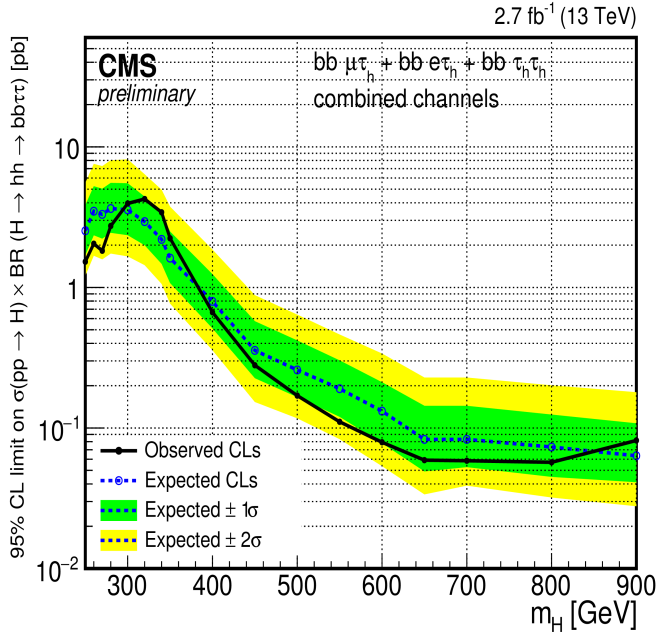

(b)

Figure 12: Observed and expected 95\% CL upper limits on cross section times branching ratio as a function of the ratio of the anomalous trilinear coupling to the SM trilinear coupling $\left(\kappa_{\lambda}=\lambda_{h h h} / \lambda_{h h h}^{S M}\right)$ combining all the final states. The red band shows the theoretical cross section expectation and its systematic uncertainty (left); Observed and expected 95\% CL upper limits on $\sigma(p p \rightarrow H) \times B R(H \rightarrow h h \rightarrow b \bar{b} \tau \tau)$ from the combination of the three channels as a function of the mass of the resonance $m_{\mathrm{H}}$.

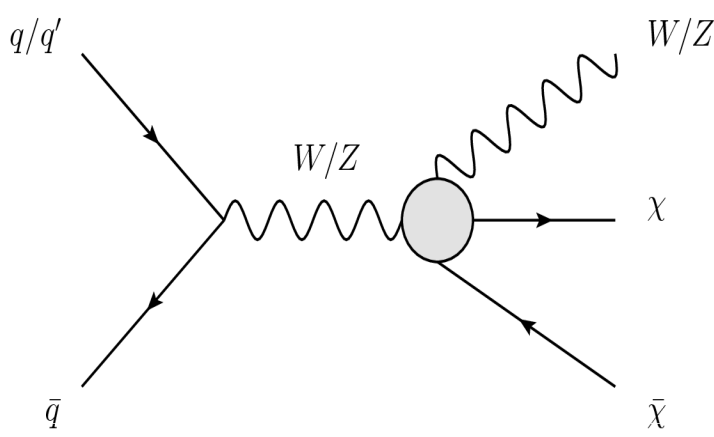

(a)

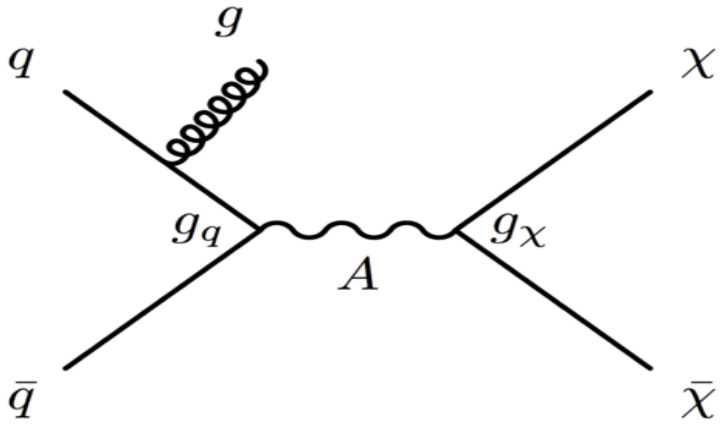

(b)

Figure 13: Typical Feynman diagrams for DM searches at LHC.

ATLAS has released results of searching for SUSY particles using Z+jets $+E_{\mathrm{T}}^{\text {miss }}$ final state, based on that $\mathrm{Z}$ bosons may be produced in the cascade decays of squarks and gluinos. The corresponding Feynman diagram for this search is shown in Figure 17.

In this search, there are 21 data events are observed and the expected background is $10.3 \pm 2.3$ (shown in 18(a)). The excess (from data) is resulting $2.2 \sigma$ deviations. However this is no obvious derivation in the same search in CMS. The searching results are interpreted in a simplified model involving the production of gluinos decaying via the second lightest neutralino to $\mathrm{Z}$ bosons and 


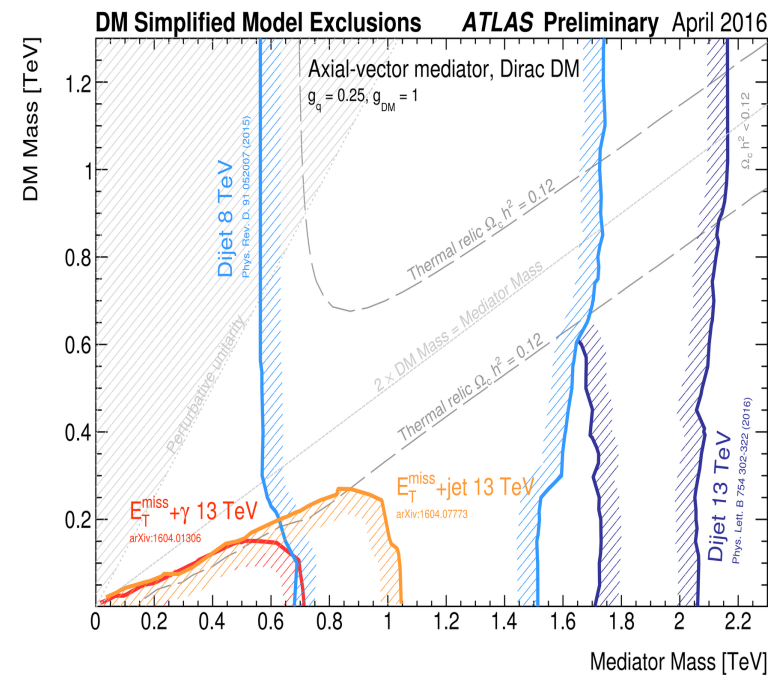

Figure 14: ATLAS DM search summary plot.

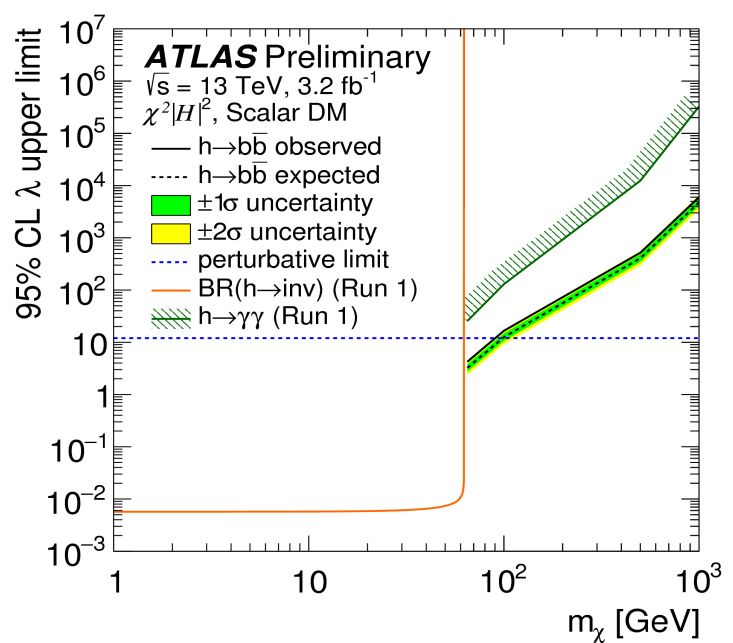

(a)

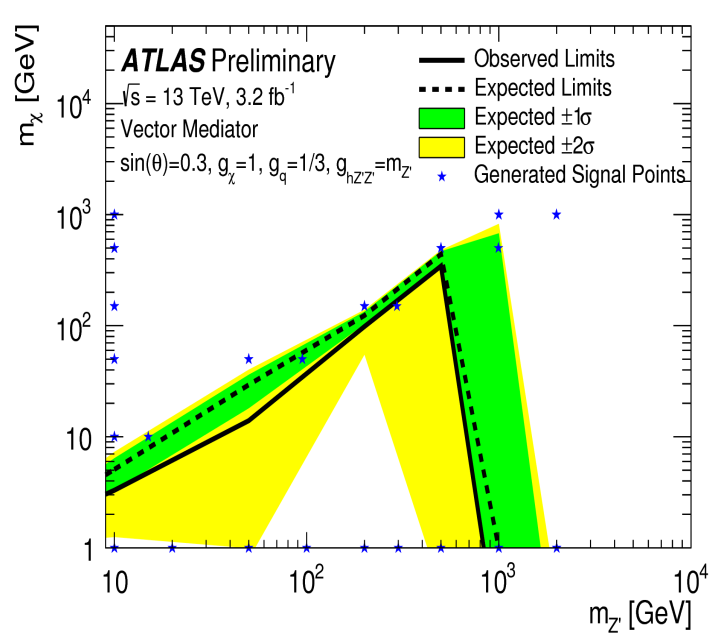

(b)

Figure 15: The interpretation of ATLAS mono-higgs $(\rightarrow b b)$ channel.

stable lightest supersymmetric particle (LSP), shown in 18(b). The gluinos are excluded up to a mass of $1.1 \mathrm{TeV}$ for a $\tilde{\chi}_{2}^{0}$ mass of $700 \mathrm{GeV}$.

The CMS search for top squark pairs decaying to all-hadronic has been presented. The corresponding Feynman diagrams are shown in Figure 19(a) and Figure 19(b). The events are categorized by the properties of reconstructed jets, the presence of top quark candidates, and $E_{\mathrm{T}}^{\mathrm{miss}}$. The categories are shown in Figure 20(a). The interpretation of this search is shown in Figure 20(b). 


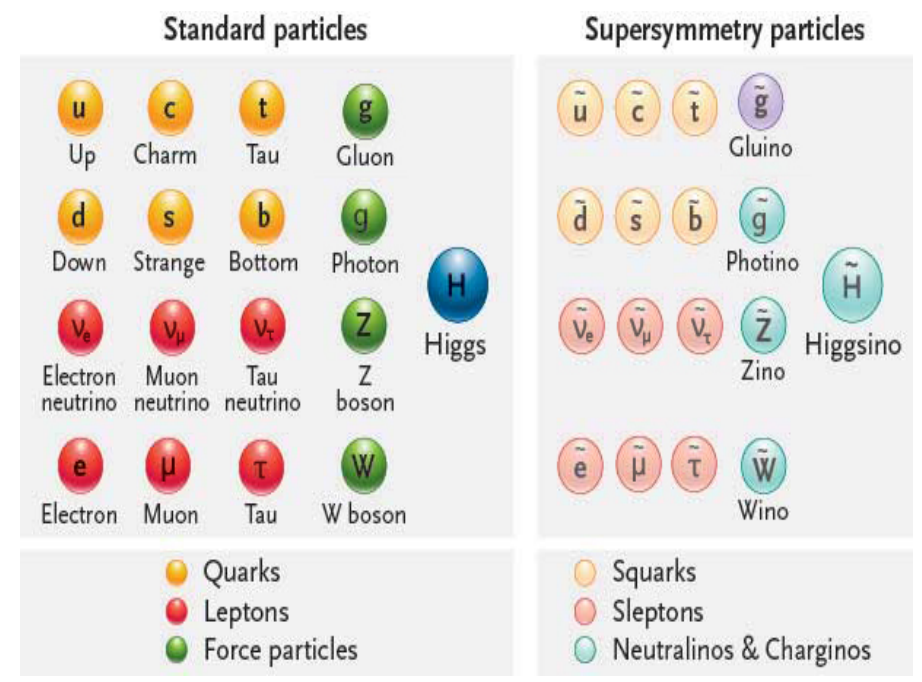

Figure 16: SM particles and their super symmetric particles.

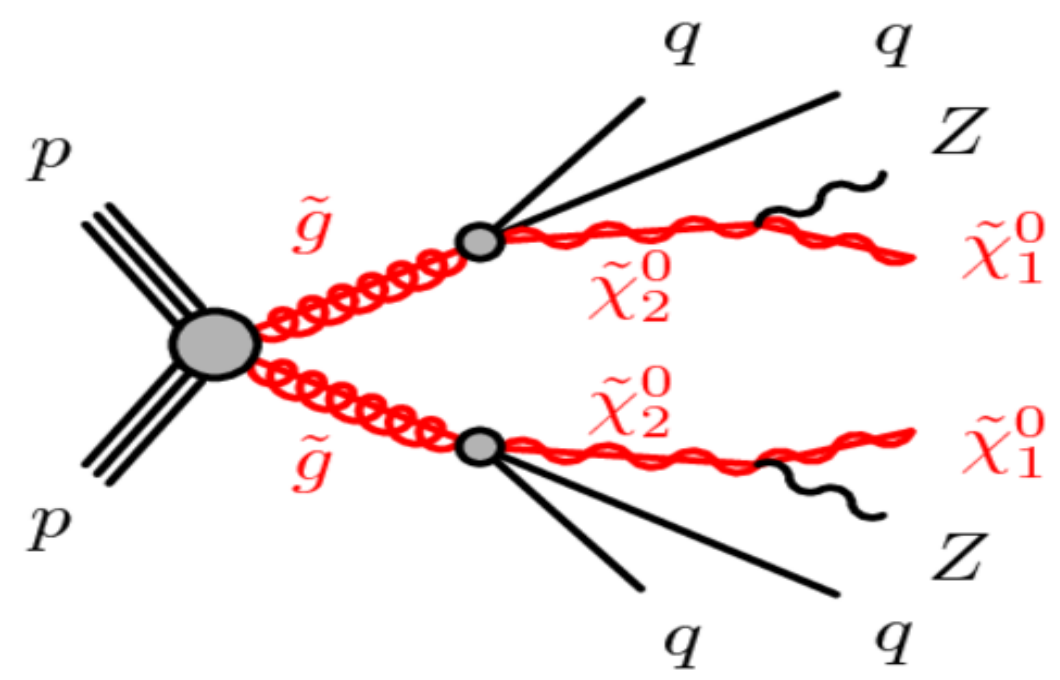

Figure 17: $\mathrm{Z}$ bosons produced in the cascade decays of squarks and gluinos.

\section{Summary}

The first $13 \mathrm{TeV}$ data have been collected by ATLAS and CMS at the LHC in year 2015. Both detectors are performing very well, provide high quality data for physics analysis. There are many new and interesting results from $201513 \mathrm{TeV}$ run are shown. They have increased the sensitivity for numerous BSM searches. With the on-going 2016 data-taking at LHC, there is high expectation from 2016 Run in the BSM searches. 


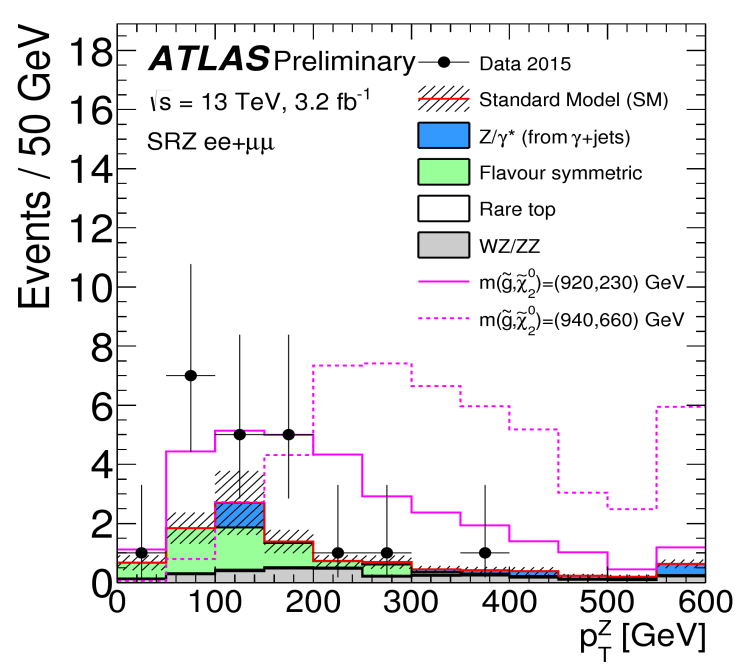

(a)

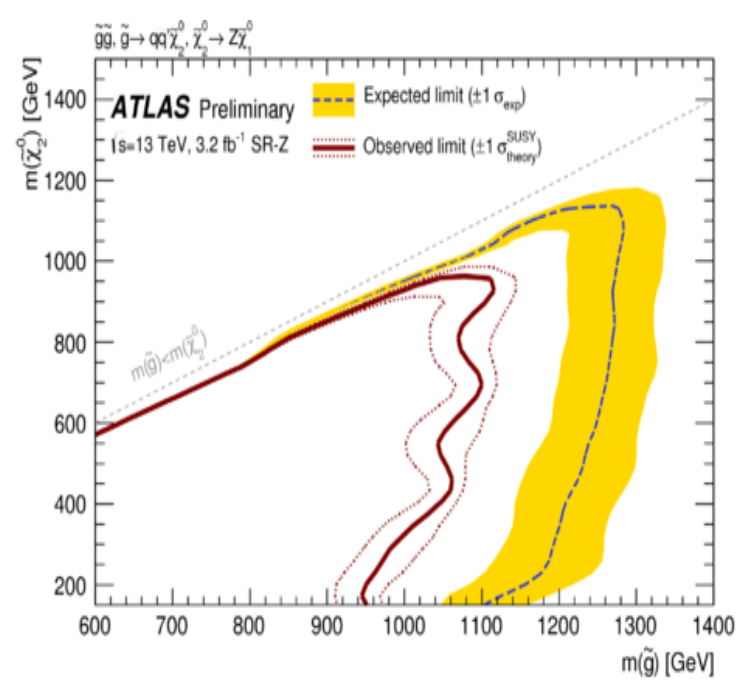

(b)

Figure 18: $p_{\mathrm{T}}^{Z}$ distribution in the signal region; The results are interpreted in a simplified model involving the production of gluinos decaying via the second lightest neutralino to $\mathrm{Z}$ bosons and stable LSP.

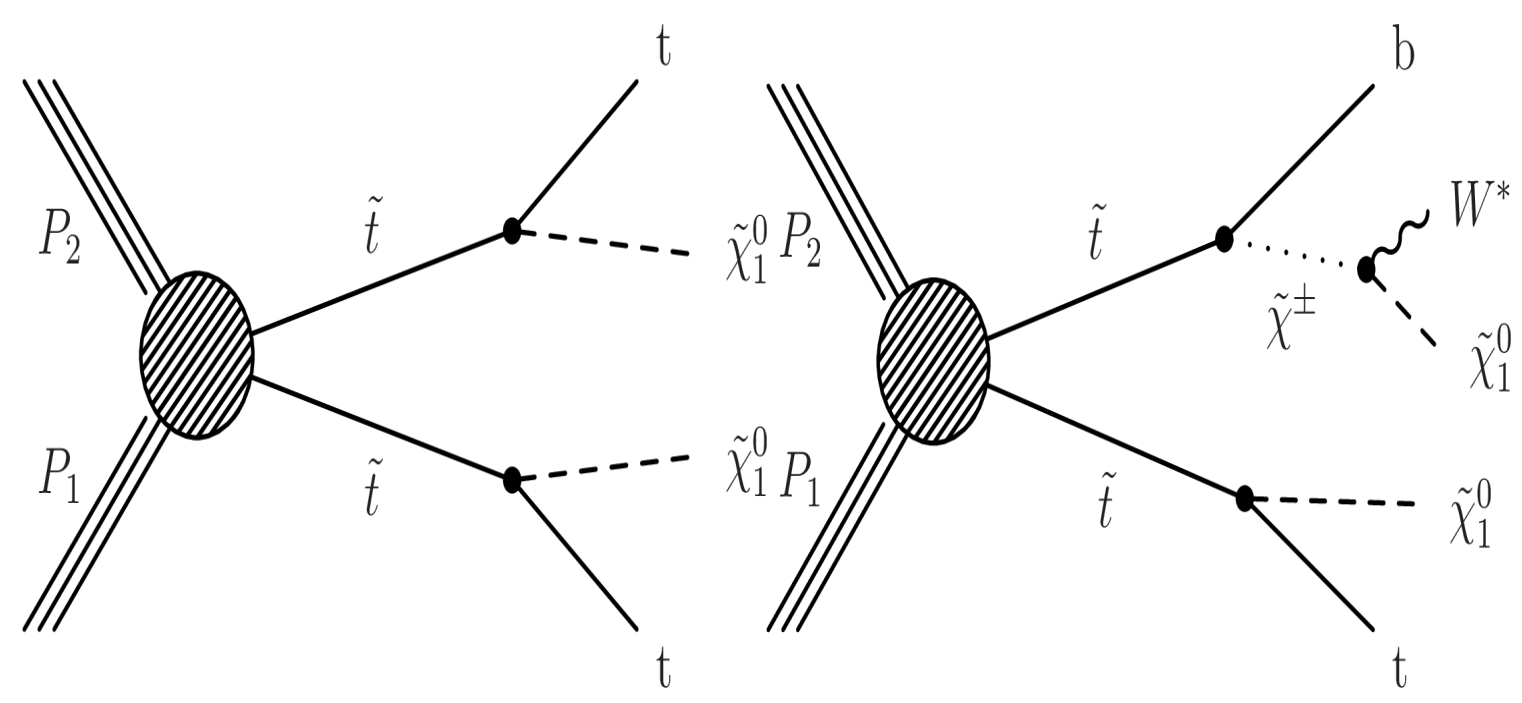

(a)

(b)

Figure 19: Top squark decay via a top quark; Top squark decaying either via a top quark or via an intermediate chargino. 


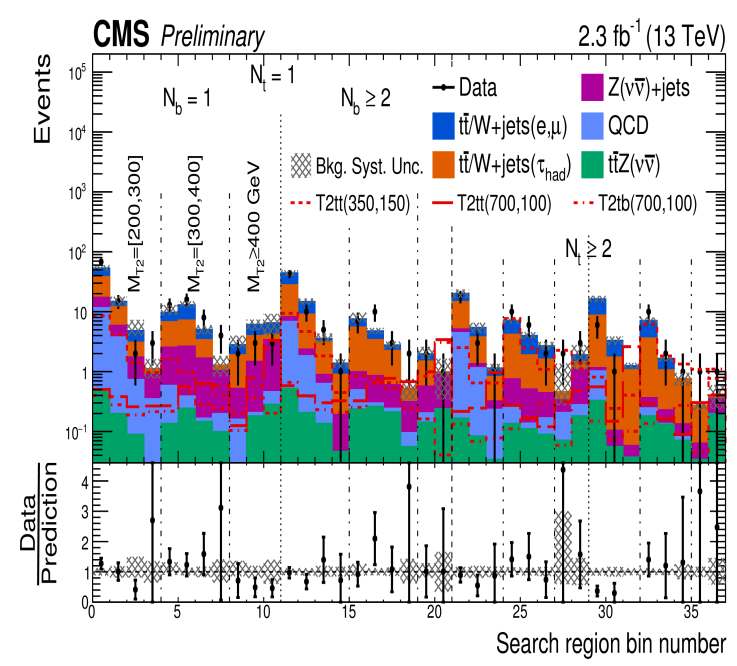

(a)

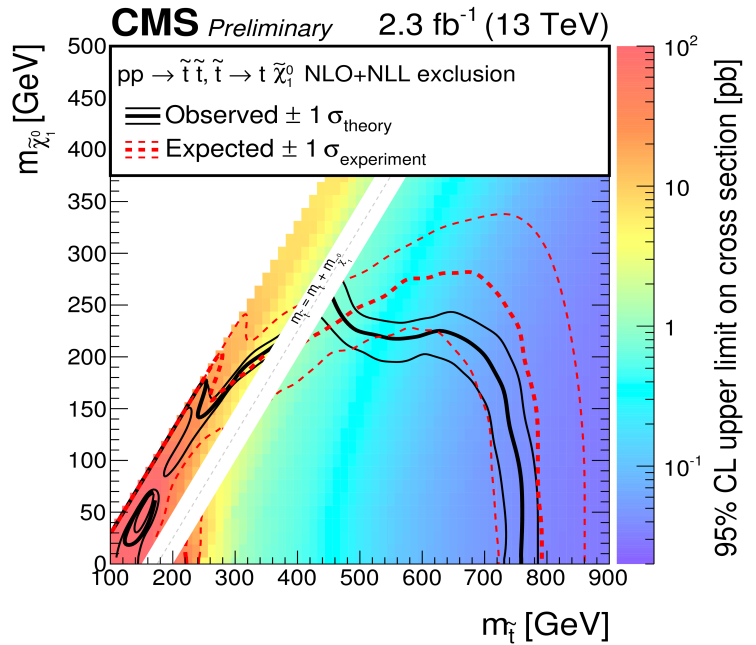

(b)

Figure 20: Event categorization and interpretation.

\section{References}

[1] CMS Collaboration, Search for massive resonances in dijet systems containing jets tagged as $\mathrm{W}$ or $\mathrm{Z}$ boson decays in $p p$ collisions at $\sqrt{s}=8 \mathrm{TeV}, 10.1007 / \mathrm{JHEP08(2014)173}$

[2] CMS Collaboration, Search for heavy neutrinos and $\mathrm{W}$ bosons with right-handed couplings in proton-proton collisions at $\sqrt{s}=8 \mathrm{TeV}, 10.1140 / \mathrm{epjc} / \mathrm{s} 10052-014-3149-\mathrm{Z}$

[3] ATLAS collaboration, Search for resonances in di-photon events with the ATLAS detector at $\sqrt{s}=13$ TeV, ATLAS-CONF-2016-018

[4] CMS Collaboration, Search for new physics in high mass di-photon events in $3.3 \mathrm{fb}^{-1}$ of proton-proton collisions at $\sqrt{s}=13 \mathrm{TeV}$ and combined interpretation of searches at $8 \mathrm{TeV}$ and 13 TeV, CMS-PAS-EXO-16-018

[5] G. Cowan, K. Cranmer, E. Gross, and O. Vitells, Asymptotic formulae for likelihood-based tests of new physics, Eur. Phys. J. C71 (2011) 1554 